Methods Retrospective data was collected on all children attending emergency department with triage complaint of chest pain using the Emergency department Information system.

Results 134 children were identified

Mean age: 11.66 years [range: $3-16 y e a r s] .80(60 \%)$ were male and $54(40 \%)$ were females. Median duration of chest pain was 2 days and half of children presented within 48 hours of pain onset.

Documentation was poor with no recorded history of cough, fever or shortness of breath in almost half of the presentations. The following specific cardiac symptoms were not recorded; radiation to arm (73.9\%), Radiation to back (76\%), Dizziness (82\%), Collapse (88\%) and Palpitations (73\%). No mention drug abuse in $100 \%$

Examination findings revealed $93 \%$ had no murmur and $99.3 \%$ had normal $\mathrm{O}_{2}$ Sats on arrival.

Investigations - ECG in $82 \%$ and was abnormal in $6 \%$. Chest $\mathrm{X}$-ray in $77 \%$ and it was abnormal in 10\%. FBC was abnormal in $<1 \%$ of patients. Troponin was done in $17 \%$ and none of these had abnormal result. D-Dimer was raised in only 2 out of 6 patients $(1.5 \%)$.

Most Common Diagnoses were Musculoskeletal 26.9\%, Costochondritis $23.1 \%$ Idiopathic $14.1 \%$ and Pneumonia $8.2 \%$. Only 1 child had cardiac diagnosis $0.8 \%$ required admission while $92 \%$ were discharged home.

Conclusions Review of 1 year of ED visits revealed that CP in children is a reasonably common complaint and is associated with tremendous anxiety and resource utilisation. There is a need for better documentation of history and examination findings. Yield from Investigations is low and should be reserved for at risk patients.

\section{G247(P) BRIDGING THE GAP BETWEEN FAMILIES, PRIMARY AND SECONDARY CARE}

doi:10.1136/archdischild-2013-304107.259

${ }^{1,2} \mathrm{C}$ Geraghty, ${ }^{1,2} \mathrm{~S}$ Banks, ${ }^{2} \mathrm{C}$ Bevan. 'Brighton and Sussex Medical School, Sussex University, Brighton, UK; ${ }^{2}$ Children's Emergency Department, Royal Alexandra Children's Hospital, Brighton, UK

Children's Emergency Department's (CED) are seeing an increasing number of primary care problems. The most worrying group are the febrile young children and the concern of a serious occult bacterial infection. We set out to analyse this group as a tool for future targeted education and support for our department, parents and primary care. Methods 100 patients aged $0-5 y e a r s$ with a temperature of $>38.0$ at presentation were reviewed. They were analysed using the febrile children NICE guidelines and assigned a risk group.LR- Low, IRIntermediate and HR High Risk.

Results (see table)

The majority $61 \%$ were LR (59\% 1-3yrs, $29 \%<1 \mathrm{yr}$ ).

$68 \%$ were self referrals. $32 \%$ were the GP referrals a third being LR and $82 \%$ were discharged home.14\% presented via ambulance, $57 \%$ were LR and $71 \%$ were discharged home.

The majority had no investigations; a third of children in the intermediate and high risk still had no investigations.

Of all diagnoses made, $46 \%$ were viral illnesses of which $74 \%$ did not receive antibiotics.

$75 \%$ were discharged home with no investigations. The younger the children and the higher the risk group, the more likely that investigations would be performed. Of the $25 \%$ requiring admission $73 \%$ had no investigations.

The majority of patients required review.

Overall the commonest diagnosis was viral infection (46\%) of which $26 \%$ still received antibiotics.

Surprisingly there were almost $100 \%$ of patients that were vaccinated.

Conclusions Parents are concerned about fever in young children and present frequently straight to CED. GPs also refer many patients with fever whom are LR category. However, in CED these
Abstract G247(P) Table 1

\begin{tabular}{|l|l|l|l|l|l|l|l|l|l|l|}
\hline & \multicolumn{3}{|c|}{$<$ year } & \multicolumn{3}{c|}{ 1-years } & \multicolumn{3}{c|}{3 years } \\
\hline Number & \multicolumn{3}{|c|}{20} & \multicolumn{3}{c|}{56} & \multicolumn{3}{c|}{15} \\
\hline Level of risk & LR & IR & HR & LR & IR & HR & LR & IR & HR \\
\hline Number & 15 & 6 & 8 & 35 & 15 & 6 & 13 & 2 & 0 \\
\hline No Investigations (\%) & $87 \%$ & $33 \%$ & $38 \%$ & $74 \%$ & $67 \%$ & $33 \%$ & $58 \%$ & $50 \%$ & 0 \\
\hline Discharged(\%) & $90 \%$ & $50 \%$ & $37 \%$ & $94 \%$ & $74 \%$ & $16 \%$ & $92 \%$ & $50 \%$ & 0 \\
\hline $\begin{array}{l}\text { Required senior } \\
\text { review }\end{array}$ & $93 \%$ & $75 \%$ & $50 \%$ & $71 \%$ & $67 \%$ & $100 \%$ & $70 \%$ & $100 \%$ & 0 \\
\hline & & & & & & & & & \\
\hline Vaccinated & $100 \%$ & $100 \%$ & $57 \%$ & $82 \%$ & $100 \%$ & $100 \%$ & $100 \%$ & $100 \%$ & 0 \\
\hline
\end{tabular}

children still require senior review although the majority no do not get investigated and are discharged. This study has helped instigate a change in practise of the local GP practises to prioritise the $<5$ year olds and to re-open the primary care unit within the hospital grounds. It has also enabled us to provide more senior cover in CED for review and timely discharge of patients without inappropriate investigations.

\section{G248(P) CAN A COMBINED TOOL WITH PAEDIATRIC ILLNESS SEVERITY ASSESSMEENT AND PAEDIATRIC EARLY WARNING SCORE BE USED AS A SAFETY TOOL FOR DISCHARGE OF PATIENTS FROM OBSERVATION AND ASSESSMENT UNIT?}

doi:10.1136/archdischild-2013-304107.260

'R Puttha, 'N Nandakumar, 'S Langworth, ${ }^{2} \mathrm{R}$ Thalava, 'L Yeung. 'Paediatrics, University Hospitals Of South Manchester, Manchester, UK; ${ }^{2}$ Orthopaedics, Tameside General Hospital NHS Foundation Trust, Manchester, UK

Aim Our aim was to evaluate if Paediatric Illness Severity Assessment (PISA) and PEWS can be used as a combined tool for safe discharge of patients from Paediatric Observation and Assessment unit (POAU).

Method We reviewed the PISA and PEWS scoring on patients attending our POAU over a busy winter on three randomised days. All patients routinely had PEWS assessed by nursing staff at the time of admission, discharge and as needed in between these two. PISA was calculated from the clinical notes. The combined tool was used to assess whether patient needed admission or discharged home. If discharged home, data was collected if there were any complications or readmissions.

Results A total of 52 patients were studied. Their age range varied from 1 day to 15 years. 37 patients were discharged home and 15 were admitted to the hospital. All of the children who were discharged had an initial PEWS score of or less than 4 or had good response with the PEWS score dropping to 0 to 2 with intervention, while their PISA grading suggested mild or moderate risk. There were no major complications in those who were discharged home. One patient was readmitted, which the parent was already cautioned. 5 patients who were admitted to the hospital had PEWS score of 0 to 2 but their PISA grading was moderate to severe risk, indicating the need for hospital admission. All those with an initial PEWS scoring above 4 or those with persistent score above 3 needed hospital admission and their PISA grading suggested moderate risk. The combined PISA and PEWS tool, in our study, when used for discharge, had a sensitivity (the probability of the child being discharged) of $100 \%$ and specificity of $97.3 \%$ with a PEWS scoring below 2 and PISA grading of mild risk. 
Conclusion The Combined tool with PISA and PEWS provides guidance in discharging patients from the observation and assessment unit. We recommend performing prospective study to validate this combined tool in a larger study population.

\section{G249(P) JOURNEY TO HEALTH: AMBULANCE USE IN PAEDIATRIC ED ATTENDERS}

doi:10.1136/archdischild-2013-304107.261

A Gite, P Heinz. Paediatrics, Cambridge University Hospital NHS Foundation Trust, Cambridge, UK

Aim To study the outcome of children brought in to the Emergency Department (ED) by ambulance.

Method All children (below16 years) who were brought in by ambulance over a period of one year to our ED were included in the study. Data was retrospectively analysed from electronic hospital records. Final diagnosis was obtained from the coding system used locally.
Results Our ED sees about 20,000 children per annum. A total of 1,828 children were brought in by ambulance between the period 01/09/2011 to 30/08/2012. Only 568 (34.88\%) children were admitted as inpatients. A total of 1,191 children were discharged home from the ED.

Diagnosis was available for 1,628 children. Out of these children $(1,628)$ where diagnosis was documented in the ED notes, trauma was the most common reason for being brought in by ambulance $502(30 \%)$ out of 1628,115 out of these were discharged. The commonest medical reason for which children were brought to ED was respiratory problems (about 22\%). Out of which 55\% were discharged. Out of 107 children brought for febrile seizure 83 (78\%) were discharged from ED. Seventy five children were diagnosed to have an upper respiratory tract infection. In total, only 50 children were admitted to paediatric intensive care unit (PICU).

Out of the 200 where diagnosis could not be found in ED records, $30(15 \%)$ were admitted and 56 were discharged, 46 children were referred to the GP in $\mathrm{ED}$ and 12 patients did not wait to see a doctor.

The number of children brought in by ambulance increased after 17:00 and decreased after 22:00.

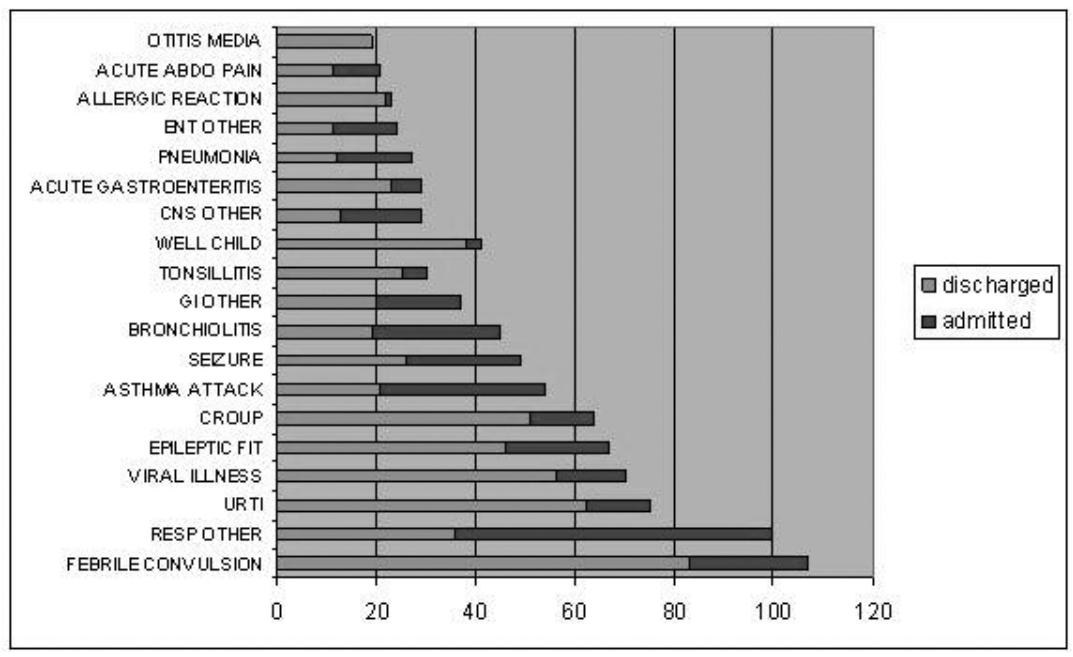

Abstract G249(P) Figure 1 Outcome of children brought in for medical reason

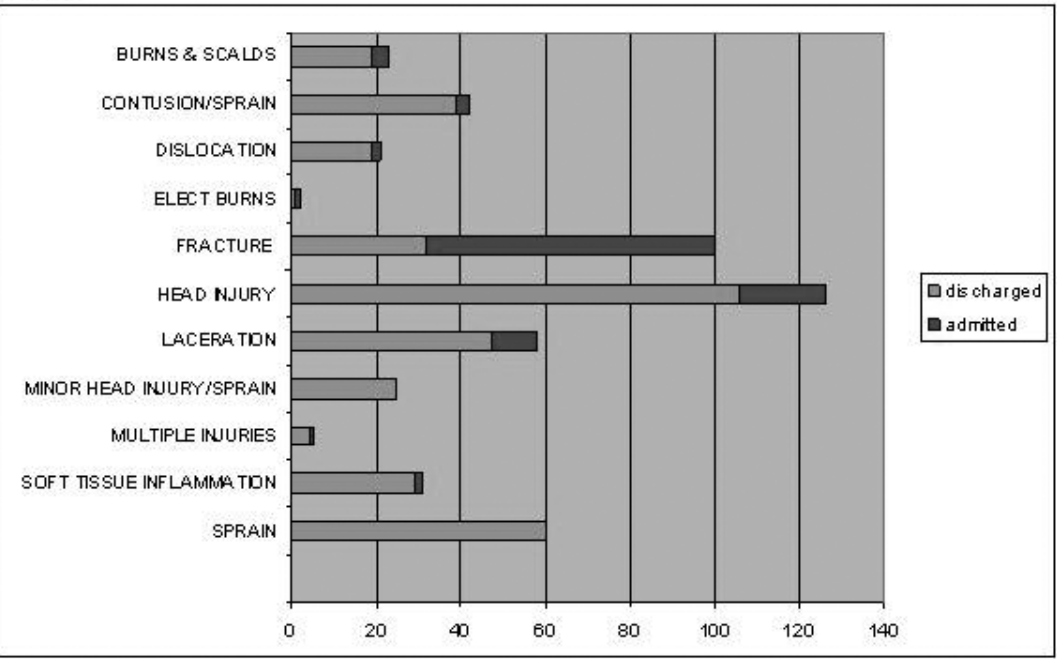

Abstract G249(P) Figure 2 Outcome of children brought in for trauma 